PROCEEDINGS OF THE

AMERICAN MATHEMATICAL SOCIETY

Volume 126, Number 9, September 1998, Pages 2641-2649

S $0002-9939(98) 04322-6$

\title{
GLOBAL ITERATION SCHEMES FOR STRONGLY PSEUDO-CONTRACTIVE MAPS
}

\author{
C. E. CHIDUME
}

(Communicated by Palle E. T. Jorgensen)

\begin{abstract}
Suppose $E$ is a real uniformly smooth Banach space, $K$ is a nonempty closed convex and bounded subset of $E$, and $T: K \rightarrow K$ is a strong pseudo-contraction. It is proved that if $T$ has a fixed point in $K$ then both the Mann and the Ishikawa iteration processes, for an arbitrary initial vector in $K$, converge strongly to the unique fixed $T$. No continuity assumption is necessary for this convergence. Moreover, our iteration parameters are independent of the geometry of the underlying Banach space and of any property of the operator.
\end{abstract}

\section{INTRODUCTION}

Let $E$ be an arbitrary real Banach space. A mapping $T$ with domain $D(T)$ and range $R(T)$ in $E$ is called a strong pseudo-contraction if there exists $t>1$ such that for all $x, y \in D(T)$ and $r>0$, the following inequality holds:

$$
\|x-y\| \leq\|(1+r)(x-y)-r t(T x-T y)\| .
$$

If $t=1$ in inequality (1) then $T$ is called pseudo-contractive. The class of pseudocontractive maps has been studied extensively by various authors (see, for example, [1], [2], [4]-[6], [7], [8], [11]-[14], [15], [16], [20], [21]-[25], [26]-[28], [29]-[30], [34]). Interest in pseudo-contractive mappings stems mainly from their firm connection with the important class of accretive operators - a mapping $U$ is called accretive if the inequality

$$
\|x-y\| \leq\|x-y+s(U x-U y)\|
$$

holds for every $x, y \in D(U)$ and for all $s>0$. If $I$ denotes the identity operator on $E$, then for $t=1$, inequality (1) implies that

$$
\|x-y\| \leq\|x-y+r[(I-T) x-(I-T) y]\|
$$

holds for all $x, y \in D(T)$ and $r>0$, so that, from inequalities (2) and (3), it follows that an operator $T$ is pseudo-contractive if and only if $(I-T)$ is accretive. Consequently, the mapping theory for accretive operators is closely related to the fixed point theory for pseudo-contractions.

Received by the editors April 22, 1996 and, in revised form, January 27, 1997.

1991 Mathematics Subject Classification. Primnary 47H17, 47H06, 47H15.

Key words and phrases. Strong pseudocontractions, accretive operators, uniformly smooth Banach spaces, duality map.

(C)1998 American Mathematical Society 
Let $E^{*}$ denote the dual space of $E$ and let $J: E \rightarrow 2^{E^{*}}$ denote the normalized duality mapping of $E$ defined by

$$
J x=\left\{x^{*} \in E^{*}:\left\langle x, x^{*}\right\rangle=\|x\| \cdot\left\|x^{*}\right\|,\left\|x^{*}\right\|=\|x\|\right\},
$$

where $\langle\cdot, \cdot\rangle$ denotes the generalized duality pairing. It is well known that if $E^{*}$ is strictly convex then $J$ is single-valued, and if $E^{*}$ is uniformly convex then $J$ is uniformly continuous on bounded subsets of $E$. In the sequel we shall denote a single-valued normalized duality map by $j$. As a consequence of a result of Kato [17], it follows from inequality (3) that $T$ is pseudo-contractive if and only if for each $x, y \in D(T)$ there exists $j(x-y) \in J(x-y)$ such that

$$
\langle(I-T) x-(I-T) y, j(x-y)\rangle \geq 0 .
$$

Furthermore, $T$ is strongly pseudo-contractive if and only if there exists $k>0$ such that

$$
\langle(I-T) x-(I-T) y, j(x-y)\rangle \geq k\|x-y\|^{2} .
$$

If $E=H$, a Hilbert space, then (4) and (5) are equivalent, respectively, to the monotonicity and strong monotonicity properties of $T$ in the sense of Minty [19].

The accretive operators were introduced independently by Browder [3] and Kato [17] in 1967. Interest in such mappings stems mainly from the fact that many physically significant problems can be modelled in terms of an initial value problem of the form

$$
\frac{d u}{d t}+T u=0, u(0)=u_{0}
$$

where $T$ is either accretive or strongly accretive in an appropriate Banach space. We observe that if $N(T)$ denotes the kernel of $T$, then members of $N(T)$ are the equilibrium points of the system (6). Consequently, considerable effort has been devoted to developing constructive techniques for the determination of the kernels of accretive operators. Moreover, since a continuous accretive operator can be approximated well by a sequence of strongly accretive ones, particular attention has been devoted to the determination of the kernels of strongly accretive maps (see for example, [1], [2], [4]-[6], [7], [8], [11]-[14], [20], [21]-[25], [26]-[28], [29], [30], $[31],[32],[34],[35])$.

Typical of the results obtained is the following theorem.

Theorem *. Let $H$ be a Hilbert space, $T: H \rightarrow H$ a bounded strongly accretive map with a nonempty kernel $N(T)$. Then the sequence $\left\{x_{n}\right\}_{n=0}^{\infty}$ defined iteratively by $x_{0} \in H$ and

$$
x_{n+1}=x_{n}-c_{n} T x_{n}, \quad n=0,1,2, \ldots,
$$

where $c_{n} \in \ell^{2} \backslash \ell^{1}$, converges globally to an element of $N(T)$.

Recall that a Banach space $E$ is called smooth if, for every $x \in E$ with $\|x\|=1$, there exists a unique $f^{*} \in E^{*}$ such that $\left\|f^{*}\right\|=f^{*}(x)=1$ (see e.g., [9, page 21]. The modulus of smoothness of $E$ is the function $\rho_{E}:[0, \infty) \rightarrow[0, \infty)$, defined by

$$
\rho_{E}(\tau)=\sup \left\{\frac{1}{2}(\|x+y\|+\|x-y\|)-1: x, y \in E,\|x\|=1,\|y\|=\tau\right\} .
$$

The Banach space $E$ is called uniformly smooth (e.g., [26]) if $\lim _{\tau \rightarrow 0} \frac{\rho_{E}(\tau)}{\tau}=0$, and for $q>1, E$ is said to be $q$-uniformly smooth if there exists a constant $c>0$ such 
that

$$
\rho_{E}(\tau) \leq c \tau^{q}, \tau \in[0, \infty)
$$

It is well known (see e.g. [26]) that

$$
L_{p}\left(\text { or } \ell_{p}\right) \text { is }\left\{\begin{array}{l}
p \text {-uniformly smooth, if } 1<p \leq 2, \\
2 \text {-uniformly smooth, if } p \geq 2 .
\end{array}\right.
$$

Various authors have extended Theorem $*$ to more general Banach spaces. Vainberg [33, pages 276-284] proved the convergence of (7) in $L_{p}$ spaces $(1<p<\infty)$ when $T$ is Lipschitz continuous and strongly accretive; the author [4] obtained the same result in $L_{p}, p \geq 2$, under less restrictive contidions. Crandall and Pazy [20] proved convergence of (7) for a continuous strongly accretive operator on an arbitrary Banach space. Reich [23], and also Liu [18], proved the convergence of (7) for an arbitrary strongly accretive operator on uniformly smooth Banach spaces. We, however, remark immediately that in these results in general Banach spaces, the conditions imposed on the iteration parameter $c_{n}$ are not convenient in applications. For instance, Crandall and Pazy [20] required that at each iteration step, $c_{k}$ be determined by

$$
c_{k}=\delta_{k+1} /\left(1+\delta_{k+1}\right),
$$

where $\delta_{k+1}=2^{-n_{k}}$ and $n_{k}$ is the least nonnegative integer such that

$$
\left\|A\left(\frac{2^{n}}{1+2^{n}} x_{k}-\frac{1}{1+2^{n}} A x_{k}\right)-A x_{k}\right\| \leq \exp \left\{-\left(\delta_{1}+\delta_{2}+\cdots+\delta_{k}+1\right)\right\} .
$$

It is clear that $c_{k}$ can hardly be determined in an explicit form from the above conditions. In [23], Reich imposed the additional assumption that $\sum_{n=0}^{\infty} c_{n}^{2}\left\|T x_{n}\right\|^{2}<\infty$. Again this condition obviously causes computational difficulties.

Recently, the author, reformulating Theorem $*$ in terms of strong pseudocontractions proved the following theorem in which the iteration parameter is easily evaluated.

Theorem C1 ([4]). Suppose $K$ is a nonempty bounded closed convex subset of $L_{p}$, $p \geq 2$, and $T: K \rightarrow K$ is a Lipschitz strong pseudo-contraction. Let $\left\{c_{n}\right\}$ be a real sequence satisfying the following conditions:

(i) $0<c_{n}<1$ for all $n \geq 1$;

(ii) $\sum_{n=1}^{\infty} c_{n}=\infty$; and

(iii) $\sum_{n=1}^{\infty} c_{n}^{2}<\infty$.

Then the sequence $\left\{x_{n}\right\}_{n=1}^{\infty}$ generated from $x_{1} \in K$ by

$$
x_{n+1}=\left(1-c_{n}\right) x_{n}+c_{n} T x_{n}, \quad n \geq 1,
$$

converges strongly to the unique fixed point of $T$.

Clearly one can choose $c_{n}=\frac{1}{n+1}$ for all $n$ in Theorem C1.

Several authors have generalized Theorem $\mathrm{C} 1$ in various directions. (See, for example, Bethke [2]; Kang [1]; Schur [29], [30]; Weng [34]; Xu et al. [28]; Xu and Roach [36].)

While most of these generalizations extend Theorem $\mathrm{C} 1$ either to more general Banach spaces or to larger classes of maps, the iteration parameters involved still depend either on the geometry of the underlying Banach space or on properties of the operator. 
For example, in his extension of Theorem $\mathrm{C} 1$ to the more general $q$-uniformly smooth Banach spaces and to the more general Ishikawa scheme (see e.g., [6] or [13]), Deng [13] required that the parameters $\alpha_{n}, \beta_{n}$ in the scheme satisfy the following conditions:

(i) $0 \leq \alpha_{n}^{s-1} \leq 2^{-1} q\left(k-L \beta_{n}-L^{2} \beta_{n}\right)\left(b L^{q}+h\right)^{-1}, n \geq 0$,

(ii) $0 \leq \beta_{n}^{s-1} \leq \min \left\{\frac{k}{2\left(L+L^{2}\right)}, \frac{q k}{\left.b L^{q}+h\right)}\right\}, n \geq 0$,

where $k$ is the constant appearing in the definition of a strongly pseudo-contractive mapping, $b$ is a constant that appears in an inequality that characterizes $q$-uniformly smooth Banach spaces, $h=\max \left\{1, \frac{q(q-1)}{2}\right\}$ and $s=\min \{2, q\}$. In [31], Tan and $\mathrm{Xu}$ extended Theorem $\mathrm{C} 1$ also to $q$-uniformly smooth Banach spaces and required that $c_{n}$ satisfy the following condition: $0<c_{n}<s_{q}, n \geq 1$, where $s_{q}$ is the (smaller) solution of the equation

$$
f(s):=q(q-1)(1-k) s-\left(1+d_{q} L^{q}\right) s^{q-1}+\frac{1}{2} q k=0, s>0
$$

(see, e.g., [31] for the meaning of the symbols). Extensions by several authors to even the more general uniformly smooth Banach spaces have also been obtained.

The most general results in this class of Banach spaces seem to be the following theorems:

Theorem R1 ([21], Theorem 1). Suppose $A$ is a strongly accretive operator with a zero, and $E$ is a uniformly smooth Banach space. Suppose that a sequence $\left\{x_{n}\right\}$ can be defined by

$$
x_{n+1} \in x_{n}-\lambda_{n} A x_{n}, \quad n \geq 0,
$$

where $x_{0} \in E$ and $\left\{\lambda_{n}\right\}$ is a positive sequence such that $\left\{\left(x_{n}-x_{n+1}\right) / \lambda_{n}\right\}$ is bounded. If $\lim _{n \rightarrow \infty} \lambda_{n}=0$ and $\sum_{n=0}^{\infty} \lambda_{n}=\infty$, then $\left\{x_{n}\right\}$ converges strongly to the zero of $A$.

Theorem C2 ([6]). Let $E$ be a real uniformly smooth Banach spae, and let $K$ be a nonempty closed convex and bounded subset of $E$. Let $T: K \rightarrow K$ be a continuous strongly pseudo-contractive mapping of $K$ into itself. Let $\left\{c_{n}\right\}_{n=1}^{\infty}$ be a real sequence satisfying the following conditions:

(i) $0<c_{n}<1$ for all $n \geq 1$,

(ii) $\sum_{n=1}^{\infty} c_{n}=\infty$, and

(iii) $\sum_{n=1}^{\infty} c_{n} b\left(c_{n}\right)<\infty$.

Then the sequence $\left\{x_{n}\right\}_{n=1}^{\infty}$ generated by $x_{1} \in K$,

$$
x_{n+1}=\left(1-c_{n}\right) x_{n}+c_{n} T x_{n}, n \geq 1,
$$

converges strongly to the unique fixed point of $T$.

While Theorems R1 and C2 extend most of the results that have appeared to either the more general uniformly smooth Banach spaces (e.g. [6], [13] and [31]) or the more general class of continuous strong pseudo-contractions, there are still several questions to answer. In particular, condition (iii) of Theorem C2, in some sense, still depends on the geometry of the underlying Banach space, while the requirement in Theorem R1 that $\left\{\left(x_{n}-x_{n+1}\right) / \lambda_{n}\right\}$ be bounded depends on a property of the operator $A$. Nevanlinna and Reich [25], however, have shown how to choose the iteration parameters to satisfy condition (iii) of Theorem $\mathrm{C} 2$ in the case $E=L_{p}(1<p<\infty)$.

It is our purpose in this paper to resolve all these problems by proving that if $E$ is a real uniformly smooth Banach space, $K$ is a nonempty closed convex 
bounded subset of $E$ and $T: K \rightarrow K$ is a strong pseudo-contraction, with a fixed point $x^{*}$ in $K$, then both the Mann and the Ishikawa iteration schemes converge strongly to $x^{*}$, for an arbitrary initial point $x_{0} \in K$. No continuity assumption is necessary for this convergence. Moreover, our iteration parameters will be totally independent both of the geometry of the underlying Banach space and of any special property of the operator. Consequently, under our setting, our theorem provides a satisfactory solution to the problem of iteratively approximating fixed points of strongly pseudo-contractive maps in real uniformly smooth Banach spaces.

\section{Preliminaries}

In the sequel, we shall make use of the following lemma.

Lemma ([22]). Let $E$ be a uniformly smooth Banach space. Then there exists a nondecreasing continuous function $b:[0, \infty) \rightarrow[0, \infty)$ satisfying the following conditions:

$$
\left\{\begin{array}{l}
\text { (i) } b(c t) \leq c b(t) \text { for all } c \geq 1 \\
\text { (ii) } \lim _{t \rightarrow 0^{+}} b(t)=0 ; \text { and } \\
\text { (iii) }\left\|x+\left.y\right|^{2} \leq\right\| x\left\|^{2}+2 \operatorname{Re}\langle y, j(x)\rangle+\max \{\|x\|, 1\} \mid y\right\| b(\mid y \|) \\
\text { for all } x, y \in E .
\end{array}\right.
$$

\section{MAin RESUlts}

We prove the following theorem.

\subsection{Convergence theorems for strongly pseudo-contractive maps.}

Theorem. Suppose $E$ is a real uniformly smooth Banach space and $K$ is a bounded closed convex and nonempty subset of $E$. Suppose $T: K \rightarrow K$ is a strongly pseudocontractive map such that $T x^{*}=x^{*}$ for some $x^{*} \in K$. Let $\left\{\alpha_{n}\right\},\left\{\beta_{n}\right\}$ be real sequences satisfying the following conditions:

(i) $0 \leq \alpha_{n}, \beta_{n}<1$ for all $n \geq 0$;

(ii) $\lim _{n \rightarrow \infty} \alpha_{n}=0 ; \lim _{n \rightarrow \infty} \beta_{n}=0$;

(iii) $\sum_{n=0}^{\infty} \alpha_{n}=\infty$.

Then, for arbitrary $x_{0} \in K$, the sequence $\left\{x_{n}\right\}$ defined iteratively by

$$
\begin{gathered}
x_{n+1}=\left(1-\alpha_{n}\right) x_{n}+\alpha_{n} T y_{n}, \\
y_{n}=\left(1-\beta_{n}\right) x_{n}+\beta_{n} T x_{n}, n \geq 0,
\end{gathered}
$$

converges strongly to $x^{*}$. Moreover, $x^{*}$ is unique.

Proof. Using (9), we compute as follows:

$$
\begin{aligned}
\| x_{n+1} & -x^{*}\left\|^{2}=\right\|\left(1-\alpha_{n}\right)\left(x_{n}-x^{*}\right)+\alpha_{n}\left(T y_{n}-x^{*}\right) \|^{2} \\
\leq & \left(1-\alpha_{n}\right)^{2}\left\|x_{n}-x^{*}\right\|^{2}+2 \alpha_{n}\left(1-\alpha_{n}\right)\left\langle T y_{n}-x^{*}, j\left(x_{n}-x^{*}\right)\right\rangle \\
& \quad+\max \left\{\left(1-\alpha_{n}\right)\left\|x_{n}-x^{*}\right\|, 1\right\} \alpha_{n}\left\|T y_{n}-x^{*}\right\| \max \left\{\left\|T y_{n}-x^{*}\right\|, 1\right\} b\left(\alpha_{n}\right) \\
\leq & \left(1-\alpha_{n}\right)^{2}\left\|x_{n}-x^{*}\right\|^{2}+M_{1} \alpha_{n} b\left(\alpha_{n}\right)+2 \alpha_{n}\left(1-\alpha_{n}\right) \delta_{n}
\end{aligned}
$$


for some constant $M_{1}>0$ (since $K$ is bounded), where

$$
\begin{aligned}
\delta_{n}: & =\left\langle T y_{n}-x^{*}, j\left(x_{n}-x^{*}\right)\right\rangle \\
& =\left\langle T y_{n}-x^{*}, j\left(x_{n}-x^{*}\right)-j\left(y_{n}-x^{*}\right)\right\rangle+\left\langle T y_{n}-T x^{*}, j\left(y_{n}-x^{*}\right)\right\rangle \\
& \leq\left\langle T y_{n}-T x^{*}, j\left(x_{n}-x^{*}\right)-j\left(y_{n}-x^{*}\right)\right\rangle+k\left\|y_{n}-x^{*}\right\|^{2} \\
& =\Delta_{n}+k\left\|y_{n}-x^{*}\right\|^{2}
\end{aligned}
$$

where $\Delta_{n}:=\left\langle T y_{n}-T x^{*}, j\left(x_{n}-x^{*}\right)-j\left(y_{n}-x^{*}\right)\right\rangle$.

Claim 1. $\Delta_{n} \rightarrow 0$ as $n \rightarrow \infty$.

Proof. Observe that $\left\{x_{n}-x^{*}\right\}$ and $\left\{y_{n}-x^{*}\right\}$ are bounded subsets of $E$, and

$$
\left\|\left(x_{n}-x^{*}\right)-\left(y_{n}-x^{*}\right)\right\|=\beta_{n}\left\|x_{n}-T x_{n}\right\| \leq(\operatorname{diam} K) \beta_{n} \rightarrow 0
$$

as $n \rightarrow \infty$. Hence, by the uniform continuity of $j$ on bounded subsets of $E$, and since $\left\{T y_{n}-T x^{*}\right\}$ is bounded, the proof of the claim is complete.

Set $M_{2}:=2\left(1-\alpha_{n}\right)$. From (12) we obtain the following estimates:

$$
\begin{aligned}
\left\|x_{n+1}-x^{*}\right\|^{2} \leq & \left(1-\alpha_{n}\right)^{2}\left\|x_{n}-x^{*}\right\|^{2}+M_{1} \alpha_{n} b\left(\alpha_{n}\right) \\
& +2 k \alpha_{n}\left(1-\alpha_{n}\right)\left\|y_{n}-x^{*}\right\|^{2}+2 \alpha_{n}\left(1-\alpha_{n}\right) \Delta_{n} \\
\leq & \left(1-\alpha_{n}\right)^{2}\left\|x_{n}-x^{*}\right\|^{2}+2 k \alpha_{n}\left(1-\alpha_{n}\right)\left\|y_{n}-x^{*}\right\|^{2} \\
& +\alpha_{n}\left[M_{2} \Delta_{n}+M_{1} b\left(\alpha_{n}\right)\right] .
\end{aligned}
$$

Now,

$$
\left\|y_{n}-x^{*}\right\|^{2} \leq\left(1-\beta_{n}\right)^{2}\left\|x_{n}-x^{*}\right\|^{2}+2 k \beta_{n}\left(1-\beta_{n}\right)\left\|x_{n}-x^{*}\right\|^{2}+M_{3} \beta_{n} b\left(\beta_{n}\right)
$$

for some constant $M_{3}>0$ (since $K$ is bounded). So,

$$
\begin{aligned}
\left\|y_{n}-x^{*}\right\|^{2} & \leq\left[1-(1-k) \beta_{n}\right]\left\|x_{n}-x^{*}\right\|^{2}+M_{3} \beta_{n} b\left(\beta_{n}\right) \\
& \leq\left\|x_{n}-x^{*}\right\|^{2}+M_{3} \beta_{n} b\left(\beta_{n}\right) .
\end{aligned}
$$

Substituting this inequality in (13) and setting $M_{4}:=2 k M_{3}\left(1-\alpha_{n}\right)$ yield the following estimates:

$$
\begin{aligned}
\| x_{n+1} & -x^{*} \|^{2} \\
\leq & \left(1-\alpha_{n}\right)^{2}\left\|x_{n}-x^{*}\right\|^{2}+2 k \alpha_{n}\left(1-\alpha_{n}\right)\left[\left\|x_{n}-x^{*}\right\|^{2}+M_{3} \beta_{n} b\left(\beta_{n}\right)\right] \\
& +\alpha_{n}\left[M_{2} \Delta_{n}+M_{1} b\left(\alpha_{n}\right)\right] \\
\leq & {\left[\left(1-\alpha_{n}\right)+k \alpha_{n}\right]^{2}\left\|x_{n}-x^{*}\right\|^{2}+M_{4} \alpha_{n} \beta_{n} b\left(\beta_{n}\right)+\alpha_{n}\left[M_{2} \Delta_{n}+M_{1} b\left(\alpha_{n}\right)\right] . }
\end{aligned}
$$

Set $J_{n}:=M_{4} \beta_{n} b\left(\beta_{n}\right)+M_{2} \Delta_{n}+M_{1} b\left(\alpha_{n}\right)$, and observe that condition (ii) and the continuity of (b) imply that $J_{n} \rightarrow 0$ as $n \rightarrow \infty$. Inequality (14) now yields that

$$
\left\|x_{n+1}-x^{*}\right\|^{2} \leq\left[1-(1-k) \alpha_{n}\right]\left\|x_{n}-x^{*}\right\|^{2}+\alpha_{n} J_{n} .
$$

Set $\lambda_{n}:=\left\|x_{n}-x^{*}\right\|^{2}, \sigma_{n}:=\alpha_{n} J_{n}$, so that the last inequality reduces to

$$
\lambda_{n+1} \leq\left[1-(1-k) \alpha_{n}\right] \lambda_{n}+\sigma_{n} .
$$

Clearly, $\left\{\lambda_{n}\right\}$ is bounded below. Let $a=\inf \left\{\lambda_{n}: n \geq 1\right\}$.

Claim 2. $a=0$. 
Proof. Assume $a \neq 0$, i.e., assume $a>0$. Then for all $n \geq 1, \lambda_{n} \geq a>0$. Observe that $\left(\sigma_{n} / \alpha_{n}\right) \rightarrow 0$ as $n \rightarrow \infty$. Hence there exists a positive integer $N_{0}$ such that for all $n \geq N_{0}, 0<\left(\sigma_{n} / \alpha_{n}\right)<a \leq \frac{1}{2}(1-k) \lambda_{n}$. This implies $\sigma_{n} \leq \frac{1}{2}(1-k) \alpha_{n} \lambda_{n}$ for all $n \geq N_{0}$. Substitute this inequality in (15) to get

$$
\begin{aligned}
0 \leq \lambda_{n+1} & \leq\left[1-(1-k) \alpha_{n}\right] \lambda_{n}+\frac{1}{2}(1-k) \alpha_{n} \lambda_{n} \\
& =\left[1-\left(\frac{1-k}{2}\right) \alpha_{n}\right] \lambda_{n} \leq \prod_{j=0}^{n}\left[1-\left(\frac{1-k}{2}\right) \alpha_{j}\right] \lambda_{j} \rightarrow 0 \text { as } n \rightarrow \infty,
\end{aligned}
$$

since $\alpha_{n} \in(0,1)$ for all $n \geq 0,\left\{\lambda_{n}\right\}$ is bounded and $\sum_{n=0}^{\infty} \alpha_{n}=\infty$, a contradiction. Hence $a=0$.

Claim 3. $\lambda_{n} \rightarrow 0$ as $n \rightarrow \infty$.

Proof. By Claim 2, there exists a subsequence $\left\{\lambda_{n_{j}}\right\}_{j=0}^{\infty}$ of $\left\{\lambda_{n}\right\}_{n=0}^{\infty}$ such that $\lambda_{n_{j}} \rightarrow$ 0 as $j \rightarrow \infty$. Now, given any $\epsilon>0$, there exists an integer $j_{*}$ sufficiently large that

$$
\frac{\sigma_{n}}{(1-k) \alpha_{n}}<\epsilon \text { and } \lambda_{n_{j_{*}}}<\epsilon \quad \forall n \geq n_{j_{*}} .
$$

Inequality (15) now yields that

$$
\lambda_{n_{j *}+1} \leq\left[1-(1-k) \alpha_{n_{j *}}\right] \epsilon+(1-k) \alpha_{n_{j *}} \epsilon=\epsilon,
$$

and a simple induction now yields $\lambda_{n_{j *}+p} \leq \epsilon$ for all integers $p \geq 1$, and this implies that $\lambda_{n} \rightarrow 0$ as $n \rightarrow \infty$. Uniqueness follows as in [4]. The proof of the theorem is complete.

Corollary. Suppose $E$ is a real uniformly smooth Banach space and $K$ is a bounded closed convex and nonempty subset of E. Suppose $T: K \rightarrow K$ is a strongly pseudocontractive map such that $T x^{*}=x^{*}$ for some $x^{*} \in K$. Let $\left\{c_{n}\right\}_{n=0}^{\infty}$ be a real sequence satisfying the following conditions:

(i) $0 \leq c_{n}<1$ for all $n \geq 0$;

(ii) $\lim _{n \rightarrow \infty} c_{n}=0$; and

(iii) $\sum_{n=0}^{\infty} c_{n}=\infty$

Then, for arbitrary $x_{0} \in K$, the sequence $\left\{x_{n}\right\}_{n=0}^{\infty}$ defined iteratively by

$$
x_{n+1}=\left(1-c_{n}\right) x_{n}+c_{n} T x_{n}, \quad n \geq 0,
$$

converges strongly to $x$. Moreover, $x^{*}$ is unique.

Proof. Set $c_{n} \equiv \alpha_{n}$ and $\beta_{n} \equiv 0$ for all $n$ in the Theorem, and the result follows immediately.

Remark 1. Our theorem is a significant generalization of most of the results that have appeared on the convergence of either the Mann or the Ishikawa iteration scheme in uniformly smooth Banach spaces. In particular, the theorem extends Theorem 2 of [13] to the more general uniformly smooth Banach spaces, to the more general class of strong pseudo-contractions with fixed points and without the dependence of the iteration parameters $\alpha_{n}, \beta_{n}$ on properties of the operator (conditions (i) and (ii) of Theorem 2 of [13]). Similarly, our theorem extends Theorem 4.2 of [31] to more general Banach spaces, more general Ishikawa schemes, a more general class of operators, and also without the dependence of the iteration parameter $\alpha_{n}$ on properties of the operator (condition (i)). Also, our theorem extends Theorem $\mathrm{C} 2$ first to the more general Ishikawa scheme, then to the more general class of operators and without the dependence of the iteration parameter $c_{n}$ on the 
geometry of the underlying Banach space (condition (iii)). Furthermore, our theorem extends Theorem R1 to the case where the iteration parameter is independent of any special property of the operator.

Remark 2. In our theorem, the iteration parameters can be chosen at the start of the iteration process. A prototype for the parameters is

$$
\alpha_{n}=\beta_{n}=c_{n}=\frac{1}{n+1} \text { for all } n \geq 0 \text {. }
$$

\section{REFERENCES}

[1] Kang Zon Bao, Iterative approximation of the solution of a locally Lipschitzian equation, Appl. Math. Mech. (English Ed.) 12(4), (1991), 409-414, translated from (Chinese) Appl. Math. Mech. 12(4), (1991), 385-389. MR 92h:47090

[2] M. Bethke, Approximation von fixpunkten streng pseudo kontritiver operatoren (Approximation of fixed points of strongly pseudo-contractive operators), Math. Naturnias Fak. 27(2), (1989), 263-270. MR 91m:47083

[3] F.E. Browder, Nonlinear mappings of nonexpansive and accretive type in Banach spaces, Bull. Amer. Math. Soc. 73 (1967), 875-882. MR 38:581

[4] C.E. Chidume, Iterative approximation of fixed points of Lipschitz strictly pseudo-contractive mappings, Proc. Amer. Math. Soc. 99 (2), (1987), 283-288. MR 87m:47122

[5] C.E. Chidume, An iterative process for nonlinear Lipschitzian strongly accretive mappings in $L_{p}$ spaces, J. Math. Anal. Appl. 151 (1990), 453-461. MR 91k:47131

[6] C.E. Chidume, Approximation of fixed points of strongly pseudo-contractive mappings, Proc. Amer. Math. Soc. 120(2), (1994), 545-551. MR 94d:47056

[7] C.E. Chidume and M.O. Osilike, Ishikawa iteration process for nonlinear Lipschitz strongly accretive mappings, J. Math. Anal. Appl. 192 (1995), 727-741. MR 96i:47099

[8] C.E. Chidume and M.O. Osilike, Fixed point iterations for strictly hemi-contractive maps in uniformly smooth Banach spaces, Numer. Funct. Anal. and Optim. 15 (7\& 8), (1994), 779-790. MR 95i: 47106

[9] I. Ciorenescu, Geometry of Banach spaces, Duality Mappings and Nonlinear Problems, Kluwer Academic Publishers, 1990. MR 91m:46021

[10] K. Deimling, Nonlinear Functional Analysis, Springer, Berlin (1985). MR 86j:47001

[11] L. Deng, On Chidume's open questions, J. Math. Anal. Appl. 174 (2), (1993), 441-449. MR 94b: 47073

[12] L. Deng, Iteration processs for nonlinear Lipschitzian strongly accretive mappings in $L_{p}$ spaces, J. Math. Anal. Appl. 188 (1994), 128-140. MR 96f:47124

[13] L. Deng, An iterative process for nonlinear Lipschitz and strongly accretive mappings in uniformly convex and uniformly smooth Banach spaces, Acta Applicande Mathematicae 32 (1993), 183-196. MR 94i:47086

[14] L. Deng and X.P. Ding, Iterative approximation of Lipschitz strictly pseudocontractive mappings in uniformly smooth Banach spaces, Nonlinear Analysis, TMA 24 (7), (1995), 981-987. MR 96a:47096

[15] J.A. Gatica and W.A. Kirk, Fixed point theorems for Lipschitz pseudo-contractive mappings, Proc. Amer. Math. Soc. 36 (1972), 111-115. MR 46:6114

[16] S. Ishikawa, Fixed point by a new iteration method, Proc. Amer. Math. Soc. 44(1), (1974), 147-150. MR 49:1243

[17] T. Kato, Nonlinear semigroups and evolution equations, J. Math. Soc. Japan 19 (1967), 508-520. MR 37:1820

[18] K. Liu, Iterative method for constructing zeros of accretive sets, M. Sc. Thesis, Xi'an Jaiotong University, 1984.

[19] G.J. Minty, Monotone (nonlinear) operators in Hilbert space, Duke Math. J. 29 (1962), 341-346. MR 29:6319

[20] M.G. Crandall and A. Pazy, On the range of accretive operators, Israel J. Math. 27 (1977), 235-246. MR 56:1142

[21] S. Reich, Constructive techniques for accretive and monotone operators, in Applied Nonlinear Analysis, (V. Lakshmikanthan, Ed.), pp. 335-345, Academic Press, New York (1979). MR 80g:47059 
[22] S. Reich, An iterative procedure for constructing zeros of accretive sets in Banach spaces, Nonlinear Anal. 2 (1978), 85-92. MR 81b:47065

[23] S. Reich, Constructing zeros of accretive operators, I, II, Appl. Anal. 8 (1979), 349-352, Appl. Anal. 9 (1979), 159-163. MR 82d:65052b; MR 82d:65052a

[24] S. Reich, Strong convergence theorems for resolvents of accretive operators in Banach spaces, J. Math. Anal. Appl. 85 (1980), 287-292. MR 82a:47050

[25] O. Nevanlinna and S. Reich, Strong convergence of contraction semigroup and of iterative methods for accretive operators in Banach spaces, Israel J. Math. 32 (1979), 44-58. MR 80e: 47057

[26] Zongben Xu and G.F. Roach, Characteristic inequalities for uniformly convex and uniformly smooth Banach spaces, J. Math. Anal. Appl. 157 (1991), 189-210. MR 92i:46023

[27] Zongben Xu and G.F. Roach, A necessary and sufficient condition for convergence of steepest descent approximation to accretive operator equations, J. Math. Anal. Appl. 167 (1992), 340354. MR 93e: 47086

[28] Zongben Xu, Zhang Bo and G.F. Roach, On the steepest descent approximation to solutions of nonlinear strongly accretive operator equations, J. Comput. Math. 7(2), 1992.

[29] J. Schu, On a theorem of C.E. Chidume concerning the iterative approximation of fixed points, Math. Nachr. 153 (1991), 313-319. MR 93b:47123

[30] J. Schu, Iterative construction of fixed points of strictly pseudo-contractive mappings, Applicable Analysis 40 (1991), 67-72. MR 92c: 47072

[31] K.K. Tan and H.K. Xu, Iterative solutions to nonlinear equations of strongly accretive operators in Banach spaces, J. Math. Anal. Appl. 178 (1993), 9-21. MR 94g:47085

[32] M.M. Vainberg, On the convergence of the process of steepest descent for nonlinear equations, Sibirsk Math. Z. 2(1961), 201-220. MR 23:A4026

[33] M.M. Vainberg, Variational method and method of monotone operators in the theory of nonlinear equations, John Wiley and Sons, 1973. MR 57:7286b

[34] Xinlong Weng, Fixed point iteration for local strictly pseudocontractive mappings, Proc. Amer. Math. Soc. 113 (1991), 727-731. MR 92b:47099

[35] H.K. Xu, Inequalities in Banach spaces with applications, Nonlinear Analysis, TMA 16(2), (1991), 1127-1138. MR 92e:47126

[36] Z. Xu and G.F. Roach, A necessary and sufficient condition for convergence of steepest descent approximation to accretive operator equations, J. Math. Anal. Appl. 167 (1992), 340-354. MR 93e:47086

[37] E. Zarantonello, Solving functional equations by constructive averaging, Tech. Report 160, US Army Research Centre, Madison, WI, 1960.

[38] E. Zarantonello, The closure of the numerical range contains the spectrum, Bull. Amer. Math. Soc. (N.S.) 70 (1964), 781-787. MR 30:3389

International Centre for Theoretical Physics, 34100 Trieste, Italy

E-mail address: chidume@ictp.trieste.it 